\title{
CARACTERIZACIÓN DE NEOPLASIAS MAMARIAS MEDIANTE ESPECTROSCOPIA DE IMPEDANCIA ELÉCTRICA: MODELO CANINO
}

\author{
William Aristizábal ${ }^{1}$ \\ María F. Eraso \\ Luis H. Alzate ${ }^{3}$ \\ Jorge $\mathrm{Cruz}^{4}$ \\ Francisco J. Pedraza ${ }^{5}$
}

\section{RESUMEN}

Introducción: La espectroscopia de impedancia eléctrica (EIE) es una técnica fácil de usar y de bajo costo que se puede utilizar para analizar tejidos biológicos en condiciones normales o patológicas. El objetivo de este trabajo fue caracterizar neoplasias de glándula mamaria benignas y malignas aplicando la técnica EIE en muestras extraídas de 45 caninos hembras (Canis lupus familiaris). Métodos: Se utilizó un medidor de impedancia eléctrica, Hioki 3532-50, para determinar los parámetros bioeléctricos: resistencia de la matriz extracelular $(\mathrm{R})$, resistencia de la matriz intracelular (S), frecuencia característica $(\mathrm{Fc})$ y capacitancia de membrana $(\mathrm{Cm})$ en un rango de frecuencias entre $42 \mathrm{~Hz}$ y $5 \mathrm{MHz}$ y se analizaron estadísticamente mediante la prueba no paramétrica U de Mann-Whitney (Wilcoxon) de dos colas. La precisión diagnóstica de la EIE se efectuó a través de curvas características de operación del receptor (COR) y tablas de doble entrada, con la histopatología como referencia. Resultados: Se encontraron diferencias estadísticamente significativas entre el tejido mamario sano y las neoplasias benignas para los parámetros R, Fc y $\mathrm{Cm}, \mathrm{p}$-value $<0,05$. Entre tejido mamario sano y neoplasias mamarias malignas se encontraron diferencias estadísticamente significativas para $\mathrm{R}$ y Fc con un p-value $<0,05$. La comparación entre lesiones tumorales benignas y malignas no presentó diferencias estadísticamente significativas, $\mathrm{p}$-value $>0,05$, para ninguna de las variables incluidas en este estudio. Conclusiones: De los parámetros analizados por EIE, la resistencia de la matriz extracelular es la que mejor permite diferenciar entre tejidos mamarios normales y neoplásicos. La EIE es una herramienta diagnóstica potencial que puede ser utilizada en la detección de cáncer mamario, con una precisión diagnóstica cercana al $80 \%$.

Palabras clave: neoplasia glándula mamaria, espectroscopia de impedancia eléctrica, resistencia matriz extracelular.

\section{CHARACTERIZATION OF MAMMARY NEOPLASIA BY ELECTRICAL IMPEDANCE SPECTROSCOPY: CANINE MODEL}

\section{ABSTRACT}

Introduction: Electrical Impedance Spectroscopy (EIS) it is an easy to use and low-cost technique that can be used to analyze biological tissues

\footnotetext{
${ }^{1}$ DEA en Bioingeniería. Departamento de Física, Facultad de Ciencias Naturales y Exactas, Universidad de Caldas. Manizales, Colombia. Correo electrónico: william.aristizabal@ucaldas.edu.co (iD ORCID: 0000-0003-0482-2805 2 MSc. Facultad de Ciencias Agropecuarias, Universidad de Caldas. Manizales, Colombia. Correo electrónico: mariafemvz@gmail.com iD ORCID: 0000-0003-4227-3616

${ }^{3}$ Ph.D. Facultad de Ciencias Naturales y Exactas, Universidad de Caldas. Manizales, Colombia. Correo electrónico: halzate@ucaldas.edu.co iD ORCID: 0000-0003-3440-6933

${ }^{4}$ Ph.D. Departamento de Producción Animal, Facultad de Ciencias Agrarias, Universidad Nacional de Colombia, sede Medellín. Medellín, Colombia. Correo electrónico: jmcrusa@unal.edu.co iD ORCID: 0000-0001-6041-9311

5 Ph.D. Departamento de Salud Animal, Facultad de Ciencias Agropecuarias, Universidad de Caldas. Manizales, Colombia. Correo electrónico: fpedraza@ucaldas.edu.co iD ORCID: 0000-0002-3217-8941
} 
in normal or pathological condition. The goal of this work was to characterize benign and malign mammary gland neoplasms applying the EIS technique in 45 female dogs (Canis lupus familiaris). Methods: An impedance meter Hioki 3532-50 was used to determine bioelectric parameters, extracellular matrix resistance $(\mathrm{R})$, intracellular matrix resistance $(S)$, characteristic frequency $(\mathrm{Cf})$, and membrane capacitance $(\mathrm{Mc})$, which were obtained in a $42 \mathrm{~Hz}$ and $5 \mathrm{MHz}$ frequencies range. Were statistically analyzed with the non-parametric test of two-tailed MannWhitney (Wilcoxon). The diagnostic precision of the test was performed using receiver operating characteristics (ROC) and two-way tables using histopathology results as reference. Results: Significant differences between healthy mammary tissue and benign neoplasms were

\section{INTRODUCCIÓN}

El cáncer mamario es una de las enfermedades más comunes en seres humanos y caninos (1). Es una de las primeras causas de muerte en mujeres de países occidentales (2) con una incidencia mundial cada vez mayor (3) estimada en 1,1 millones de nuevos casos por año (2).

En medicina veterinaria se estima una incidencia anual de 198 casos de cáncer mamario por cada 100.000 caninos (4). Este tipo de cáncer representa cerca del $50 \%$ de todas las neoplasias evidenciadas en la perra $(5,6)$, de las cuales entre el $40 \%$ y el 50\% revelan características malignas (7).

Actualmente existen numerosos y novedosos métodos que facilitan el diagnóstico, entre ellos las herramientas biológicas moleculares, no obstante, el estudio histopatológico continúa siendo uno de los más confiables y es considerado como prueba reina para la obtención del diagnóstico definitivo y clasificación tumoral, basada en las características morfológicas found for variables $\mathrm{R}, \mathrm{Cf}$ and $\mathrm{Mc}(\mathrm{p}<0.05)$. There were statistically major differences between the healthy mammary tissue and malign mammary tumors groups for $\mathrm{R}$ and $\mathrm{Cf}$ $(p<0.05)$. The comparison between malign and benign tumor lesions did not show a statistically significant difference, $p$-value $>0.05$, for any of the variables included in this study. Conclusion: Among all parameters analyzed for EIS, the extracellular matrix resistance $\mathrm{R}$ is the one that best allows differentiating between healthy and neoplastic mammary tissues. EIS is a diagnostic tool that can be used for breast cancer detection with a diagnostic precision close to $80 \%$.

Key words: neoplastic mammary gland, electrical impedance spectroscopy, extracellular matrix resistance.

del tumor (tamaño tumoral, estatus nodular, características histológicas) y actualmente se tiene en cuenta la expresión de marcadores específicos asociados a la evolución clínica (8).

La espectroscopia de impedancia eléctrica (EIE) es una técnica que consiste en aplicar una pequeña señal de corriente alterna sobre el tejido y medir la diferencia de potencial sobre el mismo. De la razón entre el voltaje leído y la corriente aplicada se puede obtener la impedancia de transferencia del tejido y los parámetros que se utilizan para caracterizarlo. Esta técnica se ha venido utilizando a nivel mundial por varios grupos de investigación para la caracterización de tejidos biológicos y especialmente para la detección de neoplasias mamarias, de cuello uterino y de próstata, entre otras $(9,10)$.

El aumento considerable de tumores mamarios en la perra, evidenciado en los últimos años, exige un rápido diagnóstico y un tratamiento eficaz con el fin de aumentar los índices de supervivencia de estos animales (11). Actualmente se investigan la espectroscopia y 
la tomografía de impedancia como métodos diagnósticos complementarios que permiten obtener resultados en tiempo real y a bajo costo $(12,13)$.

El objetivo de este trabajo fue caracterizar neoplasias de glándula mamaria benignas y malignas en 45 caninos hembras mediante la técnica de EIE, dado que es una técnica de bajo costo, no invasiva y que a futuro puede llegarse a implementar para obtener diagnósticos en tiempo real.

\section{MATERIALES Y MÉTODOS}

Se intervinieron 52 pacientes, caninos hembras (Canis lupus familiaris) de diferentes razas (Boxer, French Poodle, Labrador y Criollo) afectadas por neoplasias de glándula mamaria, atendidas en el Hospital Veterinario Diego Villegas Toro de la Universidad de Caldas (Manizales), durante el período comprendido entre noviembre de 2008 y junio de 2010. De este grupo fueron seleccionados por conveniencia 45 animales a los que se les realizó evaluación prequirúrgica, protocolo anestésico y plan terapéutico. Durante la cirugía se extrajeron muestras de tejido mamario neoplásico para su medición mediante EIE y análisis histopatológico. Se utilizó un medidor de impedancia Hioki 353250 (Hioki E.E. Corporation, Japan) para aplicar sobre el tejido una señal de corriente alterna de magnitud constante de $0,01 \mathrm{~mA}$ en un rango de frecuencias entre $42 \mathrm{~Hz}$ y $5 \mathrm{MHz}$. La corriente fue aplicada a través de una sonda tetrapolar tipo lápiz de $5,5 \mathrm{~mm}$ de diámetro, con cuatro electrodos de oro ( 2 de voltaje y 2 de corriente) de $1 \mathrm{~mm}$ de diámetro cada uno, espaciados simétricamente sobre un círculo de 1,65 mm de radio (14). El cociente entre el voltaje registrado y la corriente aplicada permite calcular la parte real e imaginaria, o la magnitud y fase de la impedancia de transferencia del tejido. En total se tomaron 304 mediciones de EIE. Con el fin de verificar la reproductibilidad, las medidas fueron tomadas por duplicado. Posteriormente se realizó la clasificación histopatológica basada en la propuesta de la Armed Forces Institute of Pathology -AFIP- (15). Con los resultados de la histopatología se conformaron tres grupos: tejidos mamarios sanos (TMS): 38 mediciones sobre 9 muestras; neoplasias mamarias benignas (NMB): 90 mediciones sobre 15 muestras; y neoplasias mamarias malignas (NMM): 176 mediciones sobre 21 muestras.

La información correspondiente a cada espectro de impedancia fue consignada en un computador portátil y mediante un programa (16) escrito en la plataforma matemática Matlab ${ }^{\circledR}$. Se realizó el ajuste de datos utilizando el método de mínimos cuadrados no lineales complejos, a una ecuación de Cole-Cole (14), que permite obtener los parámetros bioeléctricos que caracterizan las muestras de los diferentes grupos: $R 0, R \infty$ (impedancias a muy baja y muy alta frecuencia) medidas en ohmios $(\Omega)$ y la frecuencia característica -Fc- (frecuencia a la que se presenta la mayor reactancia capacitiva, parámetro relacionado con el almacenaje de cargas eléctricas en la membrana celular) medida en kilohertz $(\mathrm{kHz})$. Para la modelación eléctrica del tejido se utilizó el modelo eléctrico de Debye, el cual considera que el tejido glandular está conformado por dos ramas en paralelo: una rama que representa la resistencia de la matriz extracelular $(\mathrm{R}) \mathrm{y}$, la otra, una rama conformada por la capacitancia de membrana $(\mathrm{Cm})$ en serie con la resistencia de la matriz intracelular -S- (14). Los parámetros obtenidos mediante el ajuste al modelo de Cole-Cole (R0, R $\infty, \mathrm{Fc})$ están relacionados con $\mathrm{R}, \mathrm{S}$ y $\mathrm{Cm}$, mediante las siguientes ecuaciones:

$$
R=R_{0} ; S=R_{0} R_{\infty} / R_{0}-R_{\infty} ; F_{c}=R_{0}-R_{\infty} / 2 \pi C_{m} R_{0}^{2}
$$


Para cada medición de EIE se obtuvieron los diagramas de Bode, que se construyen con la magnitud o la fase de la impedancia en el eje de las ordenadas, y la frecuencia como variable independiente en el eje de las abscisas; y los diagramas de Nyquist, en los cuales la parte imaginaria o componente reactiva de la impedancia se grafica contra la parte real o componente resistiva de la misma. Este último diagrama permite ajustar un semicírculo con centro sobre el eje de las abscisas que corta dicho eje en los puntos $\mathrm{R} 0$ y $\mathrm{R} \infty$ respectivamente. De todas las curvas clasificadas en cada grupo, se obtuvo una curva promedio y sus parámetros bioeléctricos asociados. Los parámetros R, S, Cm y Fc fueron sometidos a la prueba de normalidad de Shapiro-Willks mediante el software estadístico Statgraphics 5.1 plus. Para establecer diferencias estadísticamente significativas entre los parámetros correspondientes a los diferentes grupos: TMS - NMM, TMS - NMB y NMB NMM, se aplicó la prueba no paramétrica $U$ de Mann-Whitney (Wilcoxon) de dos colas. Se utilizó el coeficiente de correlación de Pearson con el fin de establecer la independencia estadística entre las variables $\mathrm{R}$ y $\mathrm{S}$ en cada grupo (TMS, NMM y NMB). La precisión diagnóstica de la EIE fue evaluada con el área bajo las curvas características de operación del receptor $\operatorname{COR}(17,18)$. Para construir las tablas de doble entrada se graficaron en un mismo diagrama la sensibilidad y la especificidad en función de la resistencia de la matriz extracelular (R). Se consideró el punto de corte entra ambas curvas, ya que en este punto ambos indicadores se maximizan.

\section{RESULTADOS}

En todos los casos examinados por EIE se observó que la magnitud de la impedancia disminuye a medida que se incrementa la frecuencia (Figura 1). Este comportamiento concuerda con la modelación del tejido mediante un circuito tipo Debye $(14,19)$ en el que, a baja frecuencia, la corriente circula casi totalmente por la matriz extracelular debido a las propiedades aislantes de la membrana celular que bloquean su ingreso al citoplasma, lo que hace que la impedancia de transferencia del tejido sea alta. Con el aumento gradual de la frecuencia aumenta también la corriente que circula por el medio intracelular, debido a la disminución de la reactancia capacitiva de la membrana celular. En este estudio se observó también que a muy bajas frecuencias (entre $42 \mathrm{~Hz}$ y $1 \mathrm{kHz}$ aproximadamente) se presenta un pico en la magnitud de la impedancia que corresponde a la zona de dispersión a y en la que están involucrados fenómenos relacionados con la interfase electrodo-tejido. Esta zona de relajación que corresponde a bajas frecuencias se presenta como una curva exponencial en el sector derecho de los diagramas de Nyquist (Figura 2). Para ajustar las curvas obtenidas al modelo matemático de Cole-Cole se utilizó solo la parte semicircular del diagrama de Nyquist y se removió esta interfase.

En los diagramas de Bode (Figura 1) se puede observar que la magnitud de la impedancia de transferencia del tejido sano es mayor que las de tejidos neoplásicos benignos y malignos en un rango de frecuencias por debajo de $1 \mathrm{MHz}$, pero tiende a ser menor después de esta frecuencia. De la misma manera se comporta la parte real o componente resistiva (R) de la impedancia. Estos análisis permitieron escoger un rango de frecuencias para el ajuste de las curvas y el cálculo de los parámetros bioeléctricos que varió entre unos pocos $\mathrm{kHz}$ y $1 \mathrm{MHz}$. 


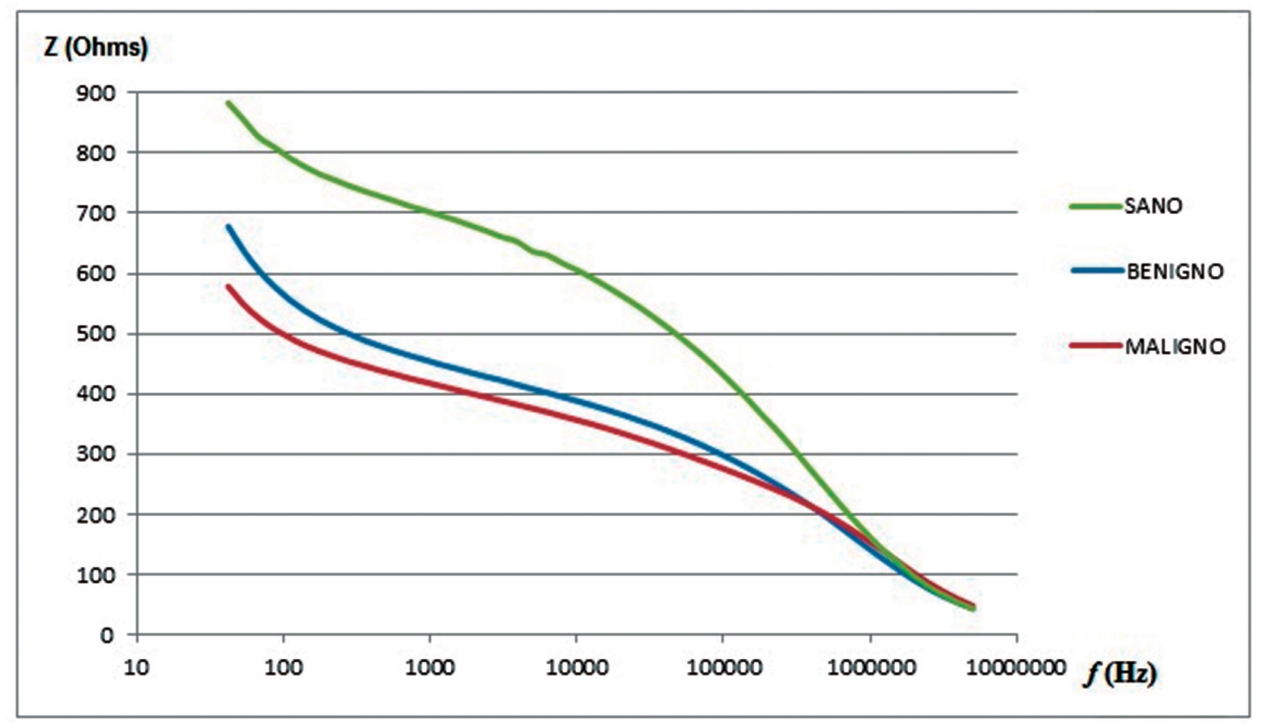

Figura 1. Diagrama de Bode de la impedancia de transferencia $\mathrm{Z}(\mathrm{Ohms})$ de los tejidos mamarios sanos y neoplásicos benignos y malignos en función de la frecuencia $(\mathrm{Hz})$, en escala logarítmica.

En los diagramas de Nyquist de la Figura 2 se observa que el semicírculo correspondiente a TMS está por encima de los semicírculos correspondientes a los tejidos que presentaron NMB y NMM. Se puede ver claramente que el semicírculo asociado al TMS difiere de manera significativa de los correspondientes a muestras de tejidos neoplásicos. A la Fc (punto máximo del semicírculo) los valores de $\mathrm{R}$ y $\mathrm{X}$ de los tejidos sanos fueron mayores que los de tejidos neoplásicos.

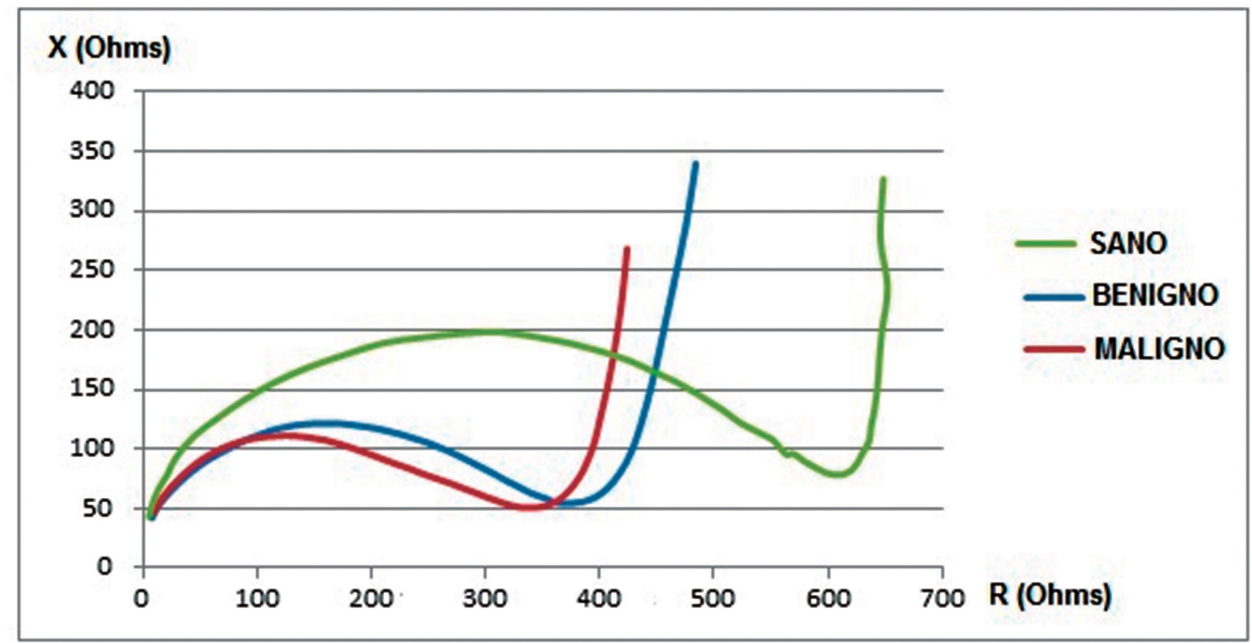

Figura 2. Diagrama de Nyquist de la parte imaginaria X (Ohms) contra la parte real $\mathrm{R}(\mathrm{Ohms})$ de la impedancia de transferencia para tejidos mamarios sanos y neoplásicos benignos y malignos. 
Tabla 1. Estadísticas descriptivas de los parámetros $\mathrm{R}, \mathrm{S}, \mathrm{Cm}, \mathrm{Fc}$ correspondientes a tejidos mamarios sanos y neoplásicos.

MEDIANA Y RANGO

MEDIAS

\begin{tabular}{|c|c|c|c|c|c|c|c|c|c|}
\hline & & Mínimo & $\begin{array}{c}\text { Percentil } \\
25\end{array}$ & Mediana & $\begin{array}{c}\text { Percentil } \\
75\end{array}$ & Máximo & Media & SD & $\begin{array}{l}\mathrm{SE} \\
\end{array}$ \\
\hline \multirow{8}{*}{ TMS } & $\mathbf{R}(\Omega)$ & 2,3 & 3,1 & 4,0 & 6,3 & 8,4 & 470,00 & 171,16 & 27,76 \\
\hline & & $\mathrm{E}+02$ & $\mathrm{E}+02$ & $\mathrm{E}+02$ & $\mathrm{E}+02$ & $\mathrm{E}+02$ & & & \\
\hline & $S(\Omega)$ & 8,28 & 4,3 & 1,14 & 2,38 & 5,40 & 14,99 & 12,92 & 2,09 \\
\hline & & E-01 & $E+00$ & $E+01$ & $E+01$ & $E+01$ & & & \\
\hline & $\mathrm{Cm}(\mathrm{nF})$ & 3,65 & 7,83 & 1,10 & 2,10 & 3,56 & 1,47 & 9,62 & 1,56 \\
\hline & & E -01 & E-01 & & & & & E-01 & E-01 \\
\hline & Fc $(\mathbf{k H z})$ & 1,1 & 1,5 & 2,9 & 6,2 & 9,1 & 367,66 & 239,07 & 38,78 \\
\hline & & $E+02$ & $E+02$ & $E+02$ & $E+02$ & $E+02$ & & & \\
\hline \multirow{6}{*}{ NMB } & $R(\Omega)$ & 96,95 & 127,02 & 228,26 & 349,20 & 951,57 & 277,03 & 180,47 & 19,02 \\
\hline & $S(\Omega)$ & 0,99 & 3,96 & 8,26 & 18,98 & 79,79 & 14,40 & 15,59 & 1,64 \\
\hline & $\mathrm{Cm}(\mathrm{nF})$ & 5,63 & 7,00 & 8,91 & 1,37 & 5,12 & 1,25 & 9,39 & 9,90 \\
\hline & & E-01 & E-01 & E-01 & & & & E-01 & E-02 \\
\hline & Fc (kHz) & 1,42 & 3,68 & 6,62 & 1,24 & 2,09 & 826,37 & 556,21 & 58,62 \\
\hline & & $\mathrm{E}+02$ & $\mathrm{E}+02$ & $\mathrm{E}+02$ & $E+03$ & $E+03$ & & & \\
\hline \multirow{8}{*}{ NMM } & $\mathbf{R}(\Omega)$ & 1,12 & 1,86 & 2,62 & 3,30 & 7,61 & 270,29 & 115,44 & 8,70 \\
\hline & & $E+02$ & $\mathrm{E}+02$ & $E+02$ & $\mathrm{E}+02$ & $E+02$ & & & \\
\hline & $S(\Omega)$ & 1,42 & 5,12 & 9,23 & 1,79 & 1,20 & 14,07 & 15,24 & 1,14 \\
\hline & & $E+00$ & $E+00$ & $E+00$ & $E+01$ & $E+02$ & & & \\
\hline & $\mathrm{Cm}(\mathrm{nF})$ & 3,50 & 5,71 & 8,04 & 1,50 & 4,05 & 1,16 & 8,56 & 6,45 \\
\hline & & E-01 & E-01 & E-01 & & & & E-01 & E-02 \\
\hline & Fc (kHz) & 1,49 & 4,17 & 7,44 & 1,24 & 2,42 & 839,16 & 527,56 & 39,76 \\
\hline & & $E+02$ & $E+02$ & $\mathrm{E}+02$ & $E+03$ & $E+03$ & & & \\
\hline
\end{tabular}

La prueba de Shapiro-Willks mostró que los parámetros $\mathrm{R}, \mathrm{S}, \mathrm{Fc}$ y $\mathrm{Cm}$, no proceden de distribuciones normales, p-value $>0,05$. Se utilizó la prueba no paramétrica U de Mann-Whitney (Wilcoxon) para comparar las medianas de los diferentes grupos.

Entre los grupos TMS y NMM se encontraron diferencias estadísticamente significativas para las variables $\mathrm{R}, \mathrm{Fc}$ y $\mathrm{Cm}$, p-value < 0,05 . El parámetro $S$ no presentó diferencias estadísticamente significativas entre estos grupos, p-value $>0,05$. Al comparar los grupos TMS y NMB se encontraron diferencias estadísticamente significativas para las variables
$\mathrm{R}$ y Fc, p-value < 0,05. Los parámetros $\mathrm{S}$ y $\mathrm{Cm}$ no presentaron diferencias estadísticamente significativas, p-value $>0,05$. La comparación entre NMB y NMM no evidenció diferencias estadísticamente significativas, p-value $>0,05$, para ninguna de las variables incluidas en este estudio.

La Cm presentó diferencia estadísticamente significativa entre TMS $(1,47+/-0,96 \mathrm{nF})$ y NMM $(1,16+/-0,85 \mathrm{nF}), \mathrm{p}$-value $=0,04$. Entre TMS y NMB $(1,25+/-0,93 \mathrm{nF})$ no se presentó diferencia estadísticamente significativa, $p$-value $>0,05$ (Tabla 1). 
La información obtenida permite deducir que la resistividad de la matriz extracelular y la frecuencia característica asociada con la componente reactiva $X$ de la impedancia bioeléctrica, son los parámetros que establecen de manera significativa diferencias entre tejido mamario normal y lesiones neoplásicas benignas y malignas. El valor promedio de R para TMS fue de $470 \Omega$, desviación estándar 171,16, coeficiente de variación $36,41 \%$. El valor promedio de $\mathrm{R}$ para NMB fue de $277,03 \Omega$, desviación estándar 180,47, coeficiente de variación 65,14\%. El valor promedio de R para NMM fue de 270,29 $\Omega$, desviación estándar 115,44, coeficiente de variación $42,71 \%$. Se puede observar cómo este parámetro disminuye con el tipo y grado de la lesión.

El valor promedio de Fc para TMS fue de 367,66 $\mathrm{kHz}$, desviación estándar 239,07, coeficiente de variación 65,02\%. Para NMB fue de 826,37 $\mathrm{kHz}$, desviación estándar 556,21, coeficiente de variación 67,30\%. Para NMM fue de 839,16 $\mathrm{kHz}$, desviación estándar 527,56, coeficiente de variación $62,86 \%$. Se puede observar cómo el valor de este parámetro aumenta con el tipo y gravedad de la lesión.

El coeficiente de correlación de Pearson mostró que en el grupo de TMB el $12,9 \%$ de las variaciones en $R$ pueden ser atribuidas a las variaciones en $\mathrm{S}$ y viceversa. En el grupo de TMM el 3,58\% y en el grupo de TMS el 3,06\%.

Al comparar los grupos TMS y NMM se encontró que el área bajo la curva COR fue 0,83, es decir, la precisión diagnóstica es del 83\% en la separación de estos dos grupos (Figura 3). Entre los grupos TMS y NMB el área bajo la curva fue 0,80, la precisión diagnóstica en este caso es del $80 \%$.

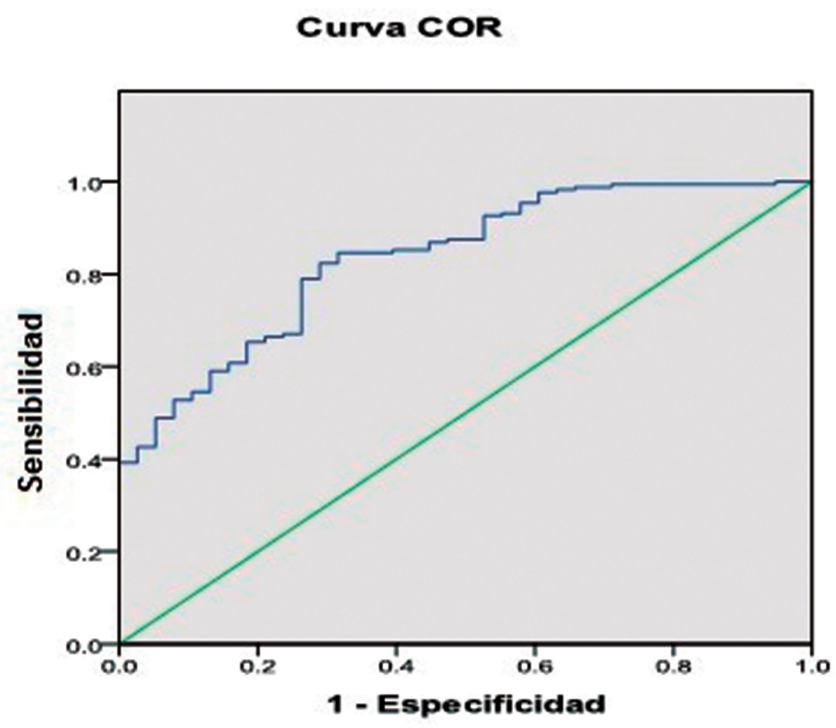

Figura 3. Curva COR para la comparación entre TMS y NMM identificada por histopatología, tomando la resistencia $(\mathrm{R})$ como indicador de impedancia eléctrica.

Entre los grupos TMS y NMB el punto de corte fue de $342 \Omega$. La sensibilidad de la prueba por EIE fue de $72,2 \%$ y la especificidad de $73,6 \%$. Entre TMS y NMM el punto de corte fue de $344 \Omega$, sensibilidad de $78,9 \%$ y especificidad de $73,6 \%$. Entre NMB y NMM el punto de corte fue de $236 \Omega$, sensibilidad de $43,2 \%$ y especificidad de $46,7 \%$. Entre NMB y TMS el valor predictivo positivo (VPP) fue de $86,7 \%$ y el valor predictivo negativo (VPN) fue de $52,8 \%$. Entre NMM y TMS el VPP fue de 93,3\% y VPN de 43,1\%. Entre NMM y NMB el VPP fue de $61,3 \%$ y el VPN de $29,6 \%$. (Ver Tabla 2 ). 
Tabla 2. Sensibilidad y especificidad de la EIE para diferenciar entre TMS y NMB, TMS y NMM, NMB y NMM, utilizando como parámetro la resistencia extracelular $(R)$.

\begin{tabular}{|c|c|c|c|c|}
\hline \multirow{5}{*}{$\begin{array}{l}\text { NMB - TMS } \\
\text { EIE: punto de } \\
\text { corte } 342 \Omega\end{array}$} & \multicolumn{4}{|c|}{ Histopatología } \\
\hline & & + & - & \\
\hline & + & 65 & 10 & 75 \\
\hline & - & 25 & 28 & 53 \\
\hline & & 90 & 38 & 128 \\
\hline \multirow{5}{*}{$\begin{array}{l}\text { NMM - TMS } \\
\text { EIE: punto de } \\
\text { corte } 344 \Omega\end{array}$} & \multicolumn{4}{|c|}{ Histopatología } \\
\hline & & + & - & \\
\hline & + & 139 & 10 & 149 \\
\hline & - & 37 & 28 & 65 \\
\hline & & 176 & 38 & 214 \\
\hline \multirow{5}{*}{$\begin{array}{l}\text { NMB - NMM } \\
\text { EIE: punto de } \\
\text { corte } 236 \Omega\end{array}$} & \multicolumn{4}{|c|}{ Histopatología } \\
\hline & & + & - & \\
\hline & + & 76 & 48 & 124 \\
\hline & - & 100 & 42 & 142 \\
\hline & & 176 & 90 & 266 \\
\hline
\end{tabular}

\section{DISCUSIÓN}

En este estudio la resistencia de la matriz extracelular fue mayor en tejidos mamarios sanos que en aquellos con neoplasias benignas y malignas, similar a los hallazgos de algunos grupos de investigación reportados por Zou y Guo (10) e investigaciones hechas por Wang et al. (20), en las que se encontraron valores significativamente diferentes de resistividad entre tejidos normales y cancerosos, con valores de impedancia baja y conductividad alta en tejidos mamarios malignos. Sin embargo, al comparar neoplasias benignas como el fibroadenoma, con neoplasias malignas, la conductividad del tumor benigno fue más alta y similar a la del tejido glandular mamario sano. Los autores afirman que el fibroadenoma tiene mejor conductividad por estar compuesto de tejido fibroso y glandular, pero está encapsulado por una membrana externa de alta resistividad que neutraliza la alta conductividad de su contenido interno. Estos resultados coinciden con los hallados en este estudio donde se observó que no hay diferencias estadísticamente significativas de los parámetros eléctricos entre las neoplasias malignas y benignas.

Una modalidad de la EIE, la técnica de espectroscopia de impedancia microeléctrica, se ha utilizado en la investigación de cultivos celulares humanos con cáncer de mama, los cuales revelaron diferencias estadísticas en la magnitud y la fase entre líneas celulares sanas y enfermas $(21,22)$; la magnitud disminuyó a medida que se incrementó la frecuencia y la fase disminuyó inicialmente, sin embargo aumentó bruscamente cuando se incrementó la frecuencia. Pese a esto, los resultados obtenidos por Han et al. (22) difieren de los obtenidos en el presente estudio, puesto que los cambios en la resistencia de los tejidos encontrados por estos investigadores fueron relativamente pequeños entre los distintos cultivos celulares.

Al contrario de los hallazgos de este estudio, la investigación realizada por Kerner et al. (23) utilizando la técnica de imágenes por 
EIE sobre tejidos in-vivo, en 26 personas con cáncer mamario, encontró que $\mathrm{R}$ y $\mathrm{S}$ en un rango de frecuencias entre 10 y $950 \mathrm{kHz}$ fueron significativamente mayores en tejido canceroso que en neoplasias benignas y en tejidos normales. Sin embargo, las mediciones de $\mathrm{Cm}$ obtenidas por estos investigadores fueron menores en tejidos cancerosos que en tejidos sanos o en neoplasias benignas, lo que coincidió con lo hallado para este parámetro en esta investigación.

De igual manera, en estudios anteriores Jossinet et al. $(24)$, Jossinet $(25,26)$ y Jossinet y Schimitt (27) analizaron tejidos mamarios ex-vivo procedentes de 60 pacientes y encontraron que la resistividad compleja, medida por EIE, presenta una aceptable precisión en la diferenciación de la respuesta de los tejidos patológicos y los tejidos normales. En la presente investigación se encontró que la EIE permite diferenciar de manera estadísticamente significativa la resistencia eléctrica de la matriz extracelular en tejidos normales y patológicos. A diferencia de los trabajos de Jossinet y colaboradores, en esta investigación la resistencia eléctrica de los TMS resultó ser mayor que las de neoplasias NMB y NMM; sin embargo, estos investigadores encontraron que los tejidos conectivos y adiposos sanos tienen una admitividad menor (mayor resistividad) que los carcinomas, los fibroadenomas, las mastopatías y las glándulas mamarias, lo que parece implicar que los resultados de la presente investigación no se diferencian de los de estos grupos de investigación y que es posible que muchas de las medidas tomadas por EIE en tejidos sanos en esta investigación fueron hechas sobre tejidos adiposos y conectivos.

Andrews et al. (28) analizaron la información procedente de 14 mujeres sospechosas de cáncer mamario. Los sensores fueron de impedancia eléctrica y espectroscopia óptica de fluorescencia, con los cuales se encontraron diferencias en la impedancia de tejidos normales y carcinomas mamarios. Estos investigadores realizaron medidas en el centro del tumor y en los bordes sanos, y encontraron que las mayores diferencias en las medidas de impedancia ocurren a la más baja frecuencia registrada $(2 \mathrm{~Hz})$. Como se observa en los diagramas de Bode, presentados en esta investigación, las mayores diferencias en la magnitud de la impedancia eléctrica y la resistencia de la matriz extracelular se presentan a bajas frecuencias, lo que coincide con los hallazgos de este grupo de la NASA.

Esta investigación permitió determinar la predisposición que tiene el tejido glandular mamario a disminuir la resistividad de la matriz extracelular a medida que el grado de malignidad aumenta. Esto es explicable desde el punto de vista biológico, ya que en enfermedades como el cáncer ocurren alteraciones histológicas y citológicas progresivas que ocasionan cambios en la organización y estructura celular, en el contenido de agua celular, en las concentraciones de electrolitos y variaciones en las propiedades de almacenamiento de carga de la membrana celular, entre otras, que conllevan a la variación en los parámetros eléctricos que caracterizan los tejidos mamarios. Resultados similares fueron encontrados en el estudio sobre las propiedades dieléctricas de tejidos mamarios en condiciones normales y patológicos, realizado en el centro de Ingeniería Biomédica en Puerto OrdazVenezuela (29).

La EIE también fue empleada por Kim et al. (30) y Jossinet y Lavandier (31) como método de análisis en mujeres con cáncer mamario mediante los diagramas de Cole-Cole, encontrando diferencias claras entre el tejido mamario normal y el tejido tumoral. Resultados similares fueron obtenidos en esta investigación, en los que se observaron diferencias claras en los diagramas de Cole-Cole correspondientes a las distintas clases de tejidos estudiados.

En este estudio la resistencia de la matriz intracelular (S) no presentó diferencia estadísticamente significativa entre TMS, 
NMB y NMM, aunque estos resultados son consistentes con el modelo utilizado, en el cual la resistencia de la $S$ debe ser mucho menor que la resistencia de la matriz extracelular $(R)$ por estar directamente relacionada con las lecturas de menor impedancia que se obtienen cuando se aumenta la frecuencia.

Se encontró que la frecuencia característica (Fc) aumenta con el grado y severidad de las lesiones, con valores mayores en NMB y NMM con respecto al TMS. Estos resultados concuerdan con los obtenidos por otros grupos de investigación quienes hallaron que la Fc es mayor en tejidos cancerosos, mediana en tejidos transitorios y baja en tejidos sanos [Stelter et al. (1998) citados en Zou y Guo (10)]. Algunos grupos de investigación han propuesto el uso de la Fc como parámetro de clasificación de tejidos mamarios sanos y con lesiones, $\mathrm{y}$ han encontrado que la $\mathrm{Fc}$ es mayor en tejidos cancerosos (mayor de $100 \mathrm{kHz}$ ) al compararlos con tejidos de mama normales (aproximadamente $20 \mathrm{kHz}$ ) (32).

El parámetro $\mathrm{Cm}$ disminuye con el grado y severidad de la lesión, pero su similitud entre TMS y NMB se puede explicar si se considera que en estas últimas las membranas celulares no sufren un deterioro tan marcado como el que se presenta en las NMM. Resultados similares se encontraron en el estudio reportado por Han et al. (22) quienes evaluaron mediante espectroscopia de impedancia 4 tipos de líneas celulares de tejido mamario. Ellos encontraron que la capacitancia de membrana fue mayor en las células del tejido mamario normal y fue disminuyendo a medida que el grado de lesión aumentaba $\left(1,94 ; 1,86 ; 1,63 ; 1,57 \mu \mathrm{F} / \mathrm{cm}^{2}\right.$ respectivamente).

La precisión diagnóstica de la EIE en la diferenciación de neoplasias benignas y malignas presentó una marcada disminución (área bajo la curva $\mathrm{COR}=0,54$ ) que puede deberse a la baja resistividad de las lesiones benignas como el fibroadenoma que, al ser afectada por la alta resistividad de la membrana exterior en la que está encapsulado, da como resultado una resistividad similar a la de los tejidos mamarios malignos. En un estudio realizado por Da Silva et al. (33), que comparó tejido mamario normal y neoplasias mamarias benignas, se concluyó que es virtualmente imposible discriminar con adecuada precisión entre tejidos con mastopatías, fibroadenomas y tejido glandular. Sin embargo, en su estudio, estos investigadores obtuvieron buenos resultados en la diferenciación entre el carcinoma mamario y los tejidos sanos.

Malich et al. (13) obtuvieron valores semejantes a los de esta investigación, utilizando la espectroscopia de impedancia eléctrica de imagen (scanning) con una sensibilidad del 79\% y una especificidad de 59\%. Sin embargo, ellos observaron la influencia de la vascularización neoplásica sobre las mediciones de conductancia, lo cual podría afectar la especificidad de la técnica, ya que a bajas frecuencias las paredes de los vasos pueden actuar potencialmente como aisladores que disminuyen la conductancia de los tejidos (13).

\section{CONCLUSIONES}

La espectroscopia de impedancia eléctrica (EIE) es una herramienta diagnóstica con potencial para ser utilizada en la detección temprana de cáncer mamario en caninos. Tiene como ventajas el bajo costo y la fácil utilización, que hacen de esta una técnica accesible y que requiere de un tiempo mínimo (menor a 5 minutos) para el procesamiento y análisis de las muestras. La obtención de resultados en tiempo real contribuye en gran medida a mejorar el pronóstico y optimizar las condiciones de vida del paciente con cáncer.

Esta técnica suministra resultados gráficos y numéricos que le permiten al investigador y al personal encargado de la salud animal clasificar con una precisión diagnóstica del 83\% un TMS de otro con NMM. Con una precisión diagnóstica 
William Aristizábal et al.

del $80 \%$ entre un TMS y otro con NMB aunque la precisión diagnóstica disminuye cuando debe diferenciar entre neoplasias malignas y benignas.

De todos los parámetros que utiliza la EIE, el parámetro bioeléctrico (R), que refleja la estructura y composición de la matriz extracelular, es el que permite diferenciar mejor entre tejidos mamarios normales y lesionados. Estos resultados permiten apreciar que la EIE tiene buena validez como prueba y puede llegar a considerarse como método de ayuda para el diagnóstico o involucrarse en estudios de tamización para este tipo de enfermedades.

Otro parámetro significativo en la EIE lo constituye la frecuencia característica, que está relacionado con la capacitancia de membrana y el producto de las resistencias extracelular e intracelular, y brinda información sobre los diferentes procesos de relajación que se presentan en los tejidos mamarios sanos y neoplásicos.

\section{REFERENCIAS}

1. Kumaraguruparan R, Prathiba D, Nagini S. Of humans and canines: Immunohistochemical analysis of PCNA, Bcl-2, p53, cytokeratin and ER in mammary tumors. Research in Veterinary Science 2006; $81(2): 218-224$.

2. Granados S, Quiles J, Gil A, Ramírez M. Lípidos de la dieta y cáncer. Nutrición Hospitalaria 2006; 21(2):44-54.

3. Vázquez T, Barrios E, Cataldi S, Vázquez A, Alonso R, Estellano F, et al. Análisis de sobrevida de una población con cáncer de mama y su relación con factores pronósticos: estudio de 1.311 pacientes seguidas durante 230 meses. Revista Médica del Uruguay 2005; 21(2):107-121.

4. Meuten DJ. Tumors in domestic animals. 4 ed. Ames, Iowa, USA: Iowa State Press; 2002.

5. Hermo G, Ripoll G, Lorenzano P, Farina H, Gabri M, Turik E, et al. Tumores de mama en la perra. Ciencia Veterinaria 2005; 7(1):1515-1883.

6. De Nardi A, Rodasky S, Sousa R, Costa T, Macedo T, Rodigheri S, et al. Prevalência de neoplasias e modalidades de tratamentos em cães, atendidos no Hospital Veterinário da Universidade Federal do Paraná. Archives of Veterinary Science 2002; 7(2):15-26.

7. Sorenmo K. Canine mammary gland tumors. The veterinary clinics small animal practice 2003; 33(3):573-596.

8. Nambiar PR, Boutin SR, Raja R, Rosenberg DW. Global gene profiling: a complement to conventional histopathological analysis of neoplasia. Veterinary Pathology 2005; 42(6):735-752.

9. Grimnes S, Martinsen O. Bioimpedance and Bioelectricity Basics. 2 ed. Great Britain: Elsevier; 2008.

10. Zou Y, Guo Z. A review of electrical impedance techniques for breast cancer detection. Medical engineering \& physics 2003; 25(2):79-90.

11. Andrade F, Figueiroa F, Bersano P, Bissacot D, Rocha N. Malignant mammary tumor in female dogs: environmental contaminants. Diagnostic Pathology 2010; 5(45):1-5.

12. Okazaki K, Tangoku A, Morimoto T, Kotani R, Yasuno E, Akutagawa M, Kinouchi Y. Basic study of a diagnostic modality employing a new impedance electrical tomography (EIT) method for noninvasive measurement in localized tissues. The journal of medical investigation 2010; 57(3-4):205-218.

13. Malich A, Böhm T, Facius M, Kleinteich I, Fleck M, Sauner D, Anderson R, Kaiser W. Electrical impedance scanning as a new imaging modality in breast cancer detection a short review of clinical value on breast application, limitations and perspectives. Nuclear instruments and methods in physics research 2003; 497(1):75-8. 
14. Brown B, Tidy J, Boston K, Blackett A, Smallwood R, Sharp F. Relation between tissue structure and imposed electrical current flow in cervical neoplasia. The Lancet 2000; 355(9207):892-895.

15. Misdorp W, Else R, Hellmén E, Lipscomb T. Histological classification of mammary tumors of the dog and the cat. 2nd series. Washington (DC): Armed Forces Institute of Pathology and World Health Organization; 1999. p. 11-25.

16. Caicedo M, Rojas J, Betancourt S, Aristizábal W, Chavarro J. Algoritmos genéticos difusos para el ajuste del modelo de cole-cole. 4to Congreso Colombiano de Computación; 2009.

17. Olarte-Echeverri G, Aristizábal-Botero W, Gallego-Sánchez PA, Rojas-Díaz J, Botero BE, Osorio GF. Detección precoz de lesiones intraepiteliales del cuello uterino en mujeres de Caldas-Colombia mediante la técnica de espectroscopia de impedancia eléctrica. Revista colombiana de obstetricia y ginecología 2007; 58(1).

18. Dawson B, Trapp R. Bioestadística médica. 4 ed. México: Manual moderno; 2005.

19. Bourne JR (ed). Bioelectrical impedance techniques in medicine. Critical Reviews in Biomedical Engineering 1996; 24(4-6).

20. Wang K, Dong X, Fu F, et al. A primary research of the relationship between breast tissues impedance spectroscopy and electrical impedance scanning. Bioinformatics and Biomedical Engineering. Or in: The 2nd International Conference on Bioinformatics and Biomedical Engineering. Shanghai. China. 2008. p. 1575-1579.

21. Han K, Han A, Frazier A. Microsystems for isolation and electrophysiological analysis of breast cancer cells from blood. Biosensors and bioelectronics 2006; 21(10):1907-1914.

22. Han A, Yang L, Frazier A. Quantification of the heterogeneity in breast cancer cell lines using wholecell impedance spectroscopy. Clinical cancer research 2007; 13(1):139-143.

23. Kerner T, Paulsen K, Hartov A, Shojo S, Poplack S. Electrical impedance spectroscopy of the breast: clinical imaging results in 26 subjects. Transactions on medical imaging 2002; 21(6):638-645.

24. Jossinet J, Lobel A, Michoudet C, Schimitt M. Quantitative technique for bioelectrical spectroscopy. Journal of Biomedical Engineering 1985; 7(4):289-294.

25. Jossinet $\mathrm{J}$. Variability of impedivity in normal and pathological breast tissue. Medical and biological engineering and computing 1996; 34(5):346-350.

26. Jossinet J. The impedivity of freshly excised human breast tissue. Physiological measurement 1998; 19(1):6175.

27. Jossinet J, Schimitt M. A review of parameters for the bioelectrical characterization of breast tissue. Annals of the New York academy of sciences 1999; 843:30-41.

28. Andrews R, Mah R, Guerrero M, Papasin R, Reed C. The NASA smart probe Project for real-time multiple microsensor tissue recognition: update. International Congress Series 2003; 1256:547-554.

29. Fariñas W, Paz Z, Orta G. Estudio del factor de disipación dieléctrica como herramienta diagnóstica. Revista Biomédica 2002; 13(4):249-255.

30. Kim B, Isaacson D, Xia H, Kao T-J, Newell JC, Saulnier G. A method for analyzing electrical impedance spectroscopy data from breast cancer patients. Physiological Measurement 2007; 28(7):S237-S246.

31. Jossinet J, Lavandier B. The discrimination of excised cancerous breast tissue samples using impedance spectroscopy. Bioelectrochemistry and bioenergetics 1998; 45(2):161-167.

32. Chauveau N, Hamzaqui L, Rochaix P, Rigaud B, Voigt J, Morucci J. Ex vivo discrimination between normal and pathological tissues in human breast surgical biopsies using bioimpedance spectroscopy. Annals of the New York Academy of Sciences 1999; 873:42-50.

33. Da Silva JE, De Sá JP, Jossinet J. Classification of breast tissue by electrical impedance spectroscopy. Medical and Biological Engineering and Computing 2000; 38(1):26-30. 